\title{
Blood pressure variability: Epidemiological and clinical issues
}

\author{
Łukasz J. Krzych, Andrzej Bochenek \\ $1^{\text {st }}$ Department of Cardiac Surgery, Medical University of Silesia, Katowice, Poland
}

\begin{abstract}
Blood pressure variability (BPV) is a classical physiological phenomenon. This paper describes major epidemiological and clinical issues of BPV which may be important to understand the background of this interesting feature. In healthy subjects, BPV is a measure of hemodynamic condition and reflects function of autonomic nervous system. BP fluctuations result from the complex interaction between environmental stimulation, genetic factors and cardiovascular control mechanisms. Abnormal BPV is recognized in persons with a blurred dipping pattern (i.e. extreme dipping, non-dipping, reverse-dipping, morning surge of BP) or increased variations of day-time or night-time BP (high BP lability). Inappropriate BPV worsens the outcome, including increase in all-cause and cardiac mortality and incidence of cardiovascular events, and advance in target organ damage. Abnormal BPV may be softened or removed with suitable time-dependent administration of anti-hypertensive agents, especially those acting on the renin-angiotensin system. (Cardiol J 2013; 20, 2: 112-120)
\end{abstract}

Key words: blood pressure variability, epidemiology, outcomes, reproducibility, pharmacotherapy

\section{Introduction}

\section{Definition and assessment of BPV}

Blood pressure (BP) is changing with every heart beat and therefore may vary periodically or accidentally during day, week or in a longer period of time, e.g. season. As BP is labile, it creates a personal condition which is defined as blood pressure variability (BPV). BPV is rather a floating physiological phenomenon than a regular hemodynamic state.

\section{Short- and long-term BPV}

There are several ways to express BPV. If $\mathrm{BPV}$ is measured continuously from beat-to-beat over an hour or up to $48 \mathrm{~h}$, it is generally called short-term variability and assessed with Fourier analysis. If BP lability is re-assessed occasionally over longer period of time (i.e. visit-to-visit with office or automatic BP measurement), it is called long-term variability. BPV may be evaluated in both normotensive and hypertensive subjects.

\section{Circadian variations in $\mathrm{BP}$}

In literature data, the most popular type of $\mathrm{BPV}$ is, however, dipping pattern of nocturnal BP. It is assessed on the basis of automatic BP measurement. To categorize $\mathrm{BP}$ it is necessary to calculate the night-day BP ratio from 12 -h recordings obtained during day and night. Four dipping categories have been already described: extreme dippers (night-day BP ratio $\leq 0.8 \rightarrow$ BP fall at night $>20 \%$ compared to day-time values), dippers (ratio $>0.8$ and $\leq 0.9 \rightarrow$ BP fall at night $\geq 10 \%$ but less than $20 \%)$, non-dippers $(0.9<$ ratio $\leq 1.0 \rightarrow$ no BP fall or BP fall at night less than 10\%) and reverse dippers (or risers) (ratio $>1.0 \rightarrow \mathrm{BP}$ rise at night) (Table 1). In many researches dipping is calculated

Address for correspondence: Ass. Prof. Łukasz Krzych, MD, PhD, $1^{\text {st }}$ Department of Cardiac Surgery, Medical University of Silesia, ul. Ziołowa 45/47, 40-635 Katowice, Poland, tel: +48 3235986 11, fax: +48 32252 70 66, e-mail: 1.krzych@wp.pl 
Table 1. Estimation of dipping pattern on the basis of day-time and night-time blood pressure (BP) values.

\begin{tabular}{|c|c|c|}
\hline $\begin{array}{l}\text { BP dipping } \\
\text { pattern }\end{array}$ & $\begin{array}{l}\text { Night-to-day } \\
\text { BP ratio }\end{array}$ & $\begin{array}{c}\text { BP fall } \\
\text { at night } \\
\text { (compared } \\
\text { to day-time) }\end{array}$ \\
\hline Extreme dippers & $\leq 0.8$ & $\geq 20 \%$ \\
\hline Dippers & $(0.8 ; 0.9]$ & $\begin{array}{c}\geq 10 \% \text { but } \\
<20 \%\end{array}$ \\
\hline Non-dippers & $(0.9 ; 1.0]$ & $\begin{array}{c}\geq 0 \% \text { but } \\
<10 \%\end{array}$ \\
\hline Reverse dippers & $>1.0$ & $\mathrm{BP}$ rise at night \\
\hline
\end{tabular}

as BP fall at night of at least 10\% (extreme dippers and dippers together) and non-dipping is recognized otherwise. This method of BPV classification is widely used in clinical studies. It is however debatable whether systolic (SBP), diastolic (DBP) or mean BP should be used to estimate the feature. But systolic value is the most common.

In epidemiological and some clinical studies, BPV may be recognized as an individual feature which creates within-person variability. Then it describes $\mathrm{BP}$ variation in one person over the definite time interval. Mean or median value is a good estimator of $\mathrm{BP}$ value and standard deviation (SD) of the mean (or interquartile range of the median) are indices of BPV. When measures are taken in an individual subject and then collected together to assess mean BP of the group, between-person (person-to-person) variability may be evaluated. Every subject adds its own BP to formulate mean (or median) value of the cohort, and SD (or interquartile range) is a measure of group BPV.

$\mathrm{BPV}$ is described either on the basis of ambulatory 24-h BP recordings or office BP measurements. Subjects with the SD below or above the mean BP of the population, measured in day-time or night-time are classified as having low or high $\mathrm{BPV}$. Interquartile range (or SD) of the median is also the acknowledged measure of variation. To combine mean and SD in one value, coefficient of variability (variation) can be calculated according to formula: $\mathrm{CV}=(\mathrm{SD}:$ mean $) \times 100 \%$.

As there are few types of BP, different types of $\mathrm{BPV}$ exist in clinical setting. In experimental studies the most popular is systolic BPV; DBP is used less frequently. For scientific purposes, we can also distinguish pulse pressure variability (as a differen- ce in systolic and diastolic BPV) or variability of mean BP (as a marker of organ tissue perfusion). Pulse pressure is proportional to stroke volume and constitutes a good marker of aortic stiffness. For that reason pulse pressure variability may become a good index of outcome in patients with advanced aortic arteriosclerosis or labile hypertension. A blunted nocturnal decrease in $\mathrm{BP}$ (non-dipping) is also independently associated with increased aortic stiffness in resistant hypertension [1].

It should be underlined that short-term and visit-to-visit $\mathrm{BPV}$, and circadian variations of $\mathrm{BP}$ (dipping pattern) are different from physiological point of view and are of different clinical importance. This review will focus on circadian variations of $\mathrm{BP}$ which include assessment of the dipping pattern and $\mathrm{BP}$ changes measured in a $24-\mathrm{h}$ period using automatic BP monitoring.

\section{Determinants of BPV}

Non-dipping pattern may be found in up to $70 \%$ of subjects [2-6]. The meta-analysis of 23 studies including 3,476 normotensive patients has revealed that the average night-to-day ratio was 0.87 for SBP and 0.83 for DBP, with ranges across the individual studies from 0.79 to 0.92 for SBP and from 0.75 to 0.9 for DBP [7].

BP fluctuations result from the complex interaction between environmental stimulation, genetic factors and cardiovascular $(\mathrm{CV})$ control mechanisms [8]. It is difficult to identify determinants of BPV because this is an individual trait. It was however found that non-dipping was more frequent is elder subjects $[9,10]$ and hypertensives, especially those untreated $[4,6,9]$. In the study enrolling 208 treated hypertensive patients [4], the incidence of non-dippers were 3.3 and 2.3 times higher in patients of $\geq 70$ and those 60-69 years of age compared to younger counterparts. Non-dipping pattern was statistically significantly 3.7 times more frequent amongst persons with a premature family history of $\mathrm{CV}$ disease. Moreover, the incidence of non-dippers was 3.0 and 4.8 times higher in overweight and obese patients compared to those with normal weight, respectively.

On the basis of measurements taken in 621 consecutive patients undergoing ambulatory $\mathrm{BP}$ monitoring at the Cleveland Clinic, Brotman et al. [11] have shown that in a statistically significant way non-dippers were older, more likely to be non-white and had higher rates of smoking, diabetes, hypertension, coronary artery disease, congestive failure and renal insufficiency. In registry data en- 
rolling 4,765 normotensive and 2,555 hypertensive subjects it has been revealed that after adjustment for potential confounders, the probability of being a non-dipper increased 2.8 times from 30 to 60 years and 5.7 times from 60 to 80 years [9]. Moreover, the risk of blunted nocturnal BP decline was 1.6-folds higher in hypertensive patients compared to normotensives. The problem of BPV is important in children and adolescents in whom early intervention helps to prevent complications of hypertension. Krzych [12] has investigated the issue in 204 consecutive children with essential hypertension. He has found that male sex increased the risk for non-dipping by 2.5 times $(\mathrm{OR}=2.45$; 95\% CI $0.87-6.87 ; \mathrm{p}=0.08)$. Gender seems to have no impact on BPV in adults.

Fava et al. [13] investigated heritability of BPV in 118 Swedish families. Nocturnal BP dipping was heritable in 38\% for SBP, 9\% for DBP and 36\% for mean BP. In another study [14], systolic and diastolic nocturnal dipping of more than $10 \%$ was heritable in $59 \%$ for SBP and $81 \%$ for diastolic BPV.

\section{Reproducibility of dipping pattern assessment}

The reproducibility of the dipping pattern has been a subject to many researches. The 4 -week reproducibility of nocturnal BP fall in untreated hypertensives was rather limited in the study of Cuspidi et al. [15]. Only $75.1 \%$ patients (including $78.7 \%$ dippers, $67.6 \%$ non-dippers and $43.2 \%$ extreme dippers) showed no change in their diurnal variations in BP. They have also showed that there were no gender and age-related differences in the repeatability of BP nocturnal fall [16]. Omboni et al. [17] have revealed that in a 4 -week period $35-40 \%$ of patients became non-dippers if they were dippers (or vice versa) regardless of treatment. In other study [18], when the first 24-h assessment of the dipping pattern was compared to the estimation on the basis of 48 -h recording, $11 \%$ hypertensive subjects switched patterns, similarly for SBP and DBP and with no difference between treated and untreated patients. In untreated patients with newly diagnosed hypertension, the nocturnal dipping pattern has been repeatable in $82 \%$ of them when re-assessed after 4 weeks [19]. Significant intra-subject variations in the diurnal fluctuations in BP have been found by Manning et al. [20] over a 12-month observation. Only $56 \%$ of normotensive and hypertensive patients had no change in their dipping status.

There are 3 explanations for those discrepancies in estimation of BPV dipping pattern.
First, BPV is a physiological phenomenon and by definition may vary over time. Second, the most appropriate method for defining day and night in published papers is not consistent. Henskens et al. [21] have proven that use of different definitions of awake-sleep and BP indices affected the classification of nocturnal BP dipping significantly, with the repeatability coefficients of no more than $48.7 \%$ and kappa values between 0.323 and 0.459 . However in the study of Verdecchia et al. [22], the area under the ROC curve of the night-to-time ratio of SBP in the assessment of $\mathrm{CV}$ events was comparable when using different clock-dependent or independent definitions of day and night. Additionally, the method of BP measurement is of some importance. Staessen et al. [9] has found that the risk of being a non-dipper was 2.4 times higher for subjects examined with auscultatory vs. oscillometric devices. Third, the impact of treatment, including pharmacotherapy on BPV is also an important point of interest.

\section{Impact of pharmacotherapy on BPV}

The potential benefit of bedtime chronotherapy with at least one hypotensive agent was investigated in the MAPEC study [23]. It has been found that despite lack of differences in ambulatory BP between groups of subjects who were randomized either to awakening or bedtime administration of medications, patients from the latter group showed higher sleep-time BP decline and reduced prevalence of non-dipping (34\% vs. $62 \%$ ). In this study, subjects taking more than 1 medication at bedtime showed significantly lower mean sleep-time BP than those ingesting all their medications upon awakening. Those differences were slightly greater for $\mathrm{SBP}(\sim 4.4 \mathrm{~mm} \mathrm{Hg})$ than $\mathrm{DBP}(\sim 4.3 \mathrm{~mm} \mathrm{Hg})$. The asleep and awake BP were lower for those who were administrated medications at bedtime [23].

Also in the study of Takeda et al. [24] bedtime administration of long-acting antihypertensive drugs helped to restore a proper dipping pattern in $71 \%$ of non-dippers. On contrary, only incomplete benefit of antihypertensive therapy on stroke reduction in older hypertensives has been documented [25]. The stroke rates were similar according to medication vs. no-medication in extreme dippers ( $12 \%$ vs. $13 \%$ ) and reverse dippers (23\% vs. $22 \%$ ), but in non-dippers there was a significantly lower rate in the medicated vs. non-medicated (4.4\% vs. $13 \%)$ groups. Also in dippers, the stroke rate was lower in the medicated compared to non-medicated $(4.7 \%$ vs. $8.8 \%)$ patients. Kario and Shimada [26] 
have revealed that after treatment with calcium channel blocker (CCB), amlodypine, the daytime $\mathrm{BP}$ was reduced in dippers, extreme dippers and non-dippers. The night-time $\mathrm{BP}$ was significantly lower only in dippers and non-dippers but not in extreme dippers. Additionally, positive correlations were found between baseline BP levels and BP reductions after treatment. In the study enrolling hypertensive subjects [4], compared with patients treated with long-acting $\mathrm{CCB}$, patients treated with angiotensin-converting enzyme inhibitors (ACE) or angiotesin II receptor blockers (ARB) alone were less frequently non-dippers $(\mathrm{OR}=0.139 ; \mathrm{p}=0.01$ ). Subjects treated with joint $\mathrm{CCB}+\mathrm{ACE}$ or $\mathrm{CCB}$ + ARB therapy had tendency of lower incidence of non-dipping ( $\mathrm{OR}=0.453 ; \mathrm{p}=0.1)$. Moreover, those treated with joint therapy including diuretics $+\mathrm{CCB}$ and diuretics $+\mathrm{ACE}$ or $\mathrm{ARB}$ were also of less frequency of non-dipping pattern: $\mathrm{OR}=0.378$; $\mathrm{p}=0.03$ and $\mathrm{OR}=0.273 ; \mathrm{p}=0.01$, respectively [4]. On the other hand, similar comparison of the effects of ACE inhibitor and CCB in hypertensives [27] has proven that $\mathrm{CCB}$ could be more effective, especially in the night-time BP reduction in non-dippers. In a prospective, randomized trial with ACE inhibitor, captopril, it was shown that at the end of the active treatment period, the prevalence of a dipping pattern in the captopril group was more than 7 times higher than in the placebo group [28]. Additionally, the night-to-day ratio and night-time BP load were significantly lower after 4 weeks of treatment with captopril administrated at bedtime.

Mathematic indices, such as trough-to-peak ratio and the smoothness index, represent useful measures of the homogeneity of the antihypertensive effect over $24 \mathrm{~h}$.

\section{Mechanism driving BPV}

Neuroendocrine mechanism are major determinants of $\mathrm{BP}$ variations responsible also for $\mathrm{BP}$ dipping pattern. At the central nervous system, integration of the major driving factors of this temporal variability is mediated by circadian rhythms of monoaminergic systems in conjunction with peripheral level, including the hypothalamic-pituitary-adrenal, hypothalamic-pituitary-thyroid, opioid, renin-angiotensin-aldosterone, endothelial systems and vasoactive peptides [29]. Those humoral secretions are typically episodic, coupled to the circadian endogenous central pacemaker clock, but have some seasonal variations. Sleep induction and arousal are influenced also by many hormones and chemical substances that exhibit 24 -h variation (e.g. arginine vasopressin, vasoactive intestinal peptide, melatonin, somatotropin, insulin, steroids, serotonin, corticotropin-releasing factor, adrenocorticotropic hormone, thyrotropin-releasing hormone, endogenous opioids, and prostaglandin $\mathrm{E} 2$ ), all with established responsiveness in the $\mathrm{CV}$ system. As a consequence, physical, mental, and pathologic stimuli that activate or inhibit neuroendocrine effectors of biological rhythmicity may also interfere with, or modify, the temporal BP structure. Moreover, immediate adjustment to exogenous components/environment demands by BP rhythms is modulated by the circadian-time-dependent alertness of biological oscillators and their neuroendocrine effectors [29].

Alteration in autonomic function plays the pivotal role in creating BPV [30] and non-dippers are characterized with an abnormal pattern of autonomic activity with higher sympathetic and lower parasympathetic modulation [31-32]. The reverse dipping state has been recognized to be related with a sympathetic activation greater for magnitude that seen in the other conditions displaying abnormalities in night-time BP pattern [33]. It has been also found that autonomic neuropathy is the pivotal factor of blunted nocturnal fall in BP in both type 1 and 2 diabetic patients [34], also in non-insulin-dependent diabetic subjects [35]. The study investigating autonomic nervous system activity in essential hypertension has documented that impaired $\mathrm{CV}$ reflexes might contribute to the decreased sympathovagal balance in non-dippers [36]. Additionally, Kario et al. [37] concluded that diurnal BP variation in elderly hypertensive patients could be associated with factors regulating circulating blood volume and impaired sensitivity of adrenergic reactivity. Night-time alpha-adrenergic blockade with doxazosine may markedly affect the nocturnal BP dipping status of hypertensives with grater reduction observed in non-dippers and risers than dippers [38].

Additional evidence is given by studies investigating the impact of physical activity on BPV. Physical activity is one of the determinants of ambulatory BP and its diurnal variations [39]. This effect has been investigated by Cavelaars et al. [40]. They have documented that non-dippers differed from dippers by an increase of vascular resistance during the night, and the degree of physical activity normally encountered during ambulatory monitoring had little influence on the diurnal BP profile or dipping status. The impact of time of day for exercise on BP dipping pattern in hypertensives has been investigated by Park et al. [41]. In this 
Table 2. Mechanisms with significant impact on altered blood pressure variations.

\begin{tabular}{|c|c|}
\hline Mechanism & Explanation \\
\hline $\begin{array}{l}\text { Autonomic nervous system } \\
\text { dysfunction }\end{array}$ & $\begin{array}{l}\text { Impaired vascular reflexes } \\
\text { Improper adrenergic stimulation }\end{array}$ \\
\hline Inflammation & $\uparrow \mathrm{C}$-reactive protein concentration \\
\hline Endothelial dysfunction & $\begin{array}{l}\text { Hemostatic imbalance ( } \uparrow \text { D-dimer, } \uparrow \text { plasminogen activator inhibitor- } 1 \text {, } \\
\uparrow \text { intercellular adhesion molecule- } 1 \text { ) }\end{array}$ \\
\hline Metabolic imbalance & $\begin{array}{l}\text { Strict relationship with the number of metabolic syndrome components } \\
\text { Hyperglycemia favors blood pressure variations }\end{array}$ \\
\hline
\end{tabular}

study, non-dippers responded to exercise irrespectively of time of day and non-dippers responded to exercise differently than dippers. Evening exercise exhibited a greater reduction in SBP at night in non-dippers than dippers and morning exercise had similar effect on day-time and $24 \mathrm{~h} \mathrm{SBP}$ reduction both in dippers and non-dippers. Furthermore, the duration of the BP reduction persisted up to $24 \mathrm{~h}$ after exercise [41].

The influence of day-time activity of BPV merits further description due to ambiguous existing researches' findings. Investigating impact of aerobic exercise on the circadian BPV, no effects were observed either for SBP or DBP in young healthy subjects [42]. In 39 untreated hypertensives, Mansoor et al. [43] found poor overall correlation between BP and activity level with marked between-subject variability in the strength of association. Moreover, almost similar results were revealed for dippers and non-dippers. On the contrary, Izdebska et al. [44] have shown that after a non-standardized 3-month moderate aerobic training, mildly hypertensive young adults had a statistically significant decrease in SBP variations compared to healthy normotensive counterparts. Those findings are in agreement with Narkiewicz et al. [45] who confirmed that there is a significant positive interaction between sympathetic traffic and day-time BPV, and in normotensive males greater $\mathrm{BP}$ decline at night was related to the greater activity during day-time.

Taking altogether, it is however clear that baseline BP condition should be taken into account in data interpretation.

The issue of inflammation and metabolic imbalance are also of great importance in the pathogenesis of abnormal BP variations. Essential hypertensive non-dippers compared to dippers have been found to exhibit higher high-sensitive C-reactive protein values [46]. Furthermore, ambulatory and nocturnal systolic BP fall interrelated and participated in the inflammatory process that accompanied non-dipping pattern. Reactive oxygen species formation was significantly increased in extreme dippers [47]. In non-dippers however interruption in hemostastic condition has also been noticed [48], with the increase in D-dimer, plasminogen activator inhibitor- 1 and intercellular adhesion molecule- 1 concentrations.

Subjects with metabolic syndrome have an increased prevalence of abnormal dipping pattern as the number of metabolic syndrome components rise [49]. Patients with metabolic syndrome are more frequent non-dippers (38.9\% vs. $24.5 \%)$ and reverse dippers (6.3\% vs. 3.3\%) and less frequently dippers (43.5\% vs. $54.4 \%$ ) or extreme dippers (11.3\% vs. $17.8 \%$ ) compared to patients without this metabolic imbalance [50]. Non-dipping pattern has been found to correlate with glucose intolerance [51]. Hyperglycemia may also be associated with abnormal diurnal BP variation [52] but interestingly, insulin resistance failed to be connected with nocturnal $\mathrm{BP}$ dipping in obese hypertensives [53] (Table 2).

\section{Clinical consequences of abnormal BP variations}

Blood pressure variations may contribute to prognosis. The dipping pattern and the night-day $\mathrm{BP}$ ratio significantly predicted mortality and $\mathrm{CV}$ events in hypertensive patients with [54] and without $[55,56]$ history of major CV disease, even after adjustment for BP values. Fagard et al. [54] have found in the former group that the outcome was worse in reverse dippers and non-dippers compared to dippers, and in the latter population [55, $56]$ the incidence of $\mathrm{CV}$ events was higher only in reverse dippers, whereas mortality was lower in extreme dippers. Additionally, the systolic night-day BP ratio independently predicted all-cause mortality and CV events. Those findings were 
confirmed regardless of gender, age and history of treatment of hypertension. Over a 10-year follow-up of persons with or without hypertension, Li et al. [57] have confirmed that the night-day BP ratio was a strong predictor of all-cause mortality but no association was found for CV events. Also in the multi-center study of Boggia et al. [58], the night-day ratio predicted mortality but not fatal combined with non-fatal events in a cohort of 7,458 subjects. In the Syst-Eur Trial of over 800 elder patients randomized to active treatment of hypertension (nitrendipine + enalapril and/or hydrochlorothiazide) or to placebo [9], CV risk increased with a higher night-day BP ratio of SBP (HR $=1.41$ per $10 \%$ increase) only in a placebo group. In the same cohort of patients, the risk of stroke increase by $80 \%$ for every $5 \mathrm{~mm} \mathrm{Hg}$ increase in night-time systolic BPV [59]. The night-day BP ratio is also a useful indicator providing significant prognostic information in patients with end-stage renal disease [60] and extreme dippers with chronic kidney disease had a 2.6-fold higher risk of CV events [61]. Rahman et al. [62] have shown that hemodialysis patients were usually non-dippers and the degree of decline in nocturnal $\mathrm{BP}$ was independently associated with left ventricle hypertrophy.

A detailed insight into the impact of circadian BP fluctuations on prognosis was the main goal of the MAPEC study. Hermida et al. [23, 63] prospectively studied 3,344 patients who were randomized into group of subject ingesting at least one hypotensive agent at bedtime or not. In the whole study population only asleep SBP was a significant predictor of outcome (any CV events, including CV morbidity and mortality). Even after adjustment for potential confounders, the CV risk decreased by $17 \%$ for every $5 \mathrm{~mm} \mathrm{Hg}$ decrease in asleep SBP. The reduced risk was confirmed for both subjects with either normal $(\mathrm{HR}=0.81)$ or elevated $\mathrm{BP}$ $(H \mathrm{R}=0.84)$. For those with high $\mathrm{BP}$, reduction in the risk was different if all medications were given on awaking ( $\mathrm{HR}=0.76$ ) or at bedtime (HR $=0.67$ ). After a 5.6 -follow-up subjects ingesting at least one BP-lowering agent at bedtime exhibited a significantly lower risk of all $\mathrm{CV}$ events $(\mathrm{RR}=0.39)$ and major events $(R R=0.33)$ than those taking all drugs upon awaking. Noteworthy, non-dippers more frequent amongst $\mathrm{CV}$ event-subjects than dippers ( $73 \%$ vs. $46 \%, \mathrm{p}<0.001)$.

A blunted reduction in nocturnal $\mathrm{BP}$ and high variations in diurnal BP may play a pivotal role in the development of target organ damage in essential hypertensive patients, including left ventricle hypertrophy and intima media thickening [64-66].
Regardless of BP values, never-treated hypertensive non-dippers have shown a significantly greater extent of target organ damage compared to dippers [67]. The measures of target organ damage were left ventricular mass index, interventricular septum thickness, left atrium and aortic root diameters. Those findings have been confirmed recently by Ivanovic et al. [68]. Patients with abnormal diurnal $\mathrm{BP}$ variation patterns (non-dippers, extreme dippers and reverse dippers) have also showed higher plasma BNP levels than those with normal BPV [69] and reverse dippers had higher microalbumin and micoglobulin excretion, and albumin/creatinine ratio [70].

Blunted reduction of systolic and diastolic night-time BP has been found to be associated with poorer cognitive performances estimated on the basis of the Mini Mental State Examination [71]. Moreover Guo et al. [72] has documented that mild cognitive impairment was more frequent in extreme dippers (32\%), non-dippers (30\%) and risers $(50 \%)$ than in dippers $(13.2 \%)$.

Furthermore, in resistant hypertension, the non-dipping pattern has been shown to be an independent predictor of $\mathrm{CV}$ mortality and the composite end point of fatal or non-fatal $\mathrm{CV}$ events, all-cause mortality and CV mortality [73, 74]. Non-dippers and reverse dippers were also at increased risk of CV events [73-75]. Also, non-dipping and reverse dipping were relatively common patterns of circadian BPV seen in acute stroke patients [76]. Additional data is given by Bastos et al. [77] who have revealed that compared to treated hypertensive subjects with no history of cerebral events, those with previous stroke or transient ischemic attack were more frequently extreme dippers ( $10.6 \%$ vs. $6.3 \%)$, non-dippers ( $48.9 \%$ vs. $41.7 \%)$ and reverse dippers (12.8\% vs. $7.6 \%)$. Moreover, patients with hypertension who suffered from any coronary event in the past were also more frequently non-dippers (56.3\% vs. $43 \%$ ) and reverse dippers (18.8\% vs. $5.9 \%$ ) [77]. CV events were more frequent also in hypertensive non-dippers aged 65 years or less [10] and in reverse dippers with or without diabetes type 2 [78]. In a 9-year follow-up study [79], type 2 diabetic patients with non-dipping pattern were at higher risk of death as compared to dippers. Non-dippers with diabetes are at increased risk of death, regardless of diabetes type [80], and the combination of non-dipping and subsequent renal impairment leads to the highest mortality rate. Reverse-dipping status in hypertensive diabetics almost 3 times $(\mathrm{HR}=2.79)$ increased the risk of $\mathrm{CV}$ events but the effect disappeared after adjustment for 24-h BP. Abnormal BP dipping 
status has been documented to increase risk of death or hospitalization for heart failure exacerbation [81] and non-dipping pattern has been associated with an over 2-times higher risk of congestive heart failure in initially healthy Finish male [82]. Finally, non-dippers with sustained hypertension have been found to be at a 2 -fold higher risk of developing atrial fibrillation than dippers [83].

On the other hand, no association between nocturnal decline in BP and mortality has been found in the Ohasama cohort study [84]. Although both nocturnal hypotension and erratic diastolic BPV assessed by Fourier spectral analysis predicted mortality, subsequent study of the PAMELA data has failed to prove any relationship between the risk of death and 24-h, day-time and night-time BPV expressed as SDs [85]. Also in the study of Hansen et al. [86, 87] recruiting subjects from 11 populations, there was no prognostic value of reading-to-reading BPV and in the study of Gavish et al. [88] there was no ability of the BPV to predict the outcome on the basis of the ratio of 24-h ambulatory systolic BPV to diastolic variability.

Finally, recent findings designate that the mean nocturnal $\mathrm{BP}$ value is the most sensitive predictor of cardio- and cerebrovascular morbidity, and mortality [89-90]. Moreover, regardless of the relative circadian BP values, insufficient BP fall at night compared to day-time level or a high night to day $\mathrm{BP}$ ratio is associated with poor prognosis $[89,90]$.

\section{Conclusions}

Blood pressure variability is a classical physiological phenomenon as BP varies individually continuously and episodically. In healthy subjects, $\mathrm{BPV}$ is a measure of hemodynamic condition and reflects function of autonomic nervous system. BP fluctuations result from the complex interaction between environmental stimulation, genetic factors and $\mathrm{CV}$ control mechanisms. Abnormal BPV is recognized in persons with a blurred dipping pattern (i.e. extreme dipping, non-dipping, reverse-dipping, morning surge of $\mathrm{BP}$ ) or increased variations of day-time or nigh-time BP (high BP lability). Inappropriate $\mathrm{BP}$ decline at night worsens the outcome, including increase in all-cause and cardiac mortality and incidence of $\mathrm{CV}$ events, and advance in target organ damage. Abnormal BPV may be softened or removed with suitable time-dependent administration of anti-hypertensive agents, especially those acting on the renin-angiotensin system.

Conflict of interest: none declared

\section{References}

1. Castelpoggi CH, Pereira VS, Fiszman R, Cardoso CR, Muxfeldt ES, Salles GF. A blunted decrease in nocturnal blood pressure is independently associated with increased aortic stiffness in patients with resistant hypertension. Hypertens Res, 2009; 32: 591-596.

2. Loimaala A, Turjanmaa V, Vuori I, Oja P, Pasanen M, Uusitalo A. Variation of ambulatory blood pressure in healthy middle-aged men. J Hum Hypertens, 1997; 11: 227-231.

3. Ragot S, Herpin D. Abnormalities in the circadian rhythm of arterial blood pressure. Physiopathological mechanism and clinical implications. Arch Mal Coeur Vaiss, 1999; 92: 1745-1753.

4. Qiu YG, Yao XY, Tao QM et al. Profile on circadian blood pressure and the influencing factors in essential hypertensive patients after treatment. Zhonghua Liu Xing Bing Xue Za Zhi, 2004; 25: 710-714.

5. Gorostidi M, Sobrino J, Segura J et al. Ambulatory blood pressure monitoring in hypertensive patients with high cardiovascular risk: A cross-sectional analysis of a 20,000-patient database in Spain. J Hypertens, 2007; 25: 977-984.

6. de la Sierra A, Redon J, Banegas JR et al. Prevalence and factors associated with circadian blood pressure patterns in hypertensive patients. Hypertension, 2009; 53: 466-472.

7. Staessen J, Guo C, De Cort P et al. Mean and range of the ambulatory pressure in normotensive subjects. Chin Med J (Engl), 1992; 105: 328-333.

8. Parati G, Faini A, Valentini M. Blood pressure variability: Its measurement and significance in hypertension. Curr Hypertens Rep, 2006; 8: 199-204.

9. Staessen JA, Bieniaszewski L, O'Brien E et al. Nocturnal blood pressure fall on ambulatory monitoring in a large international database. The “Ad Hoc' Working Group. Hypertension, 1997; 29: 30-39.

10. Di Iorio A, Marini E, Lupinetti M, Zito M, Abate G. Blood pressure rhythm and prevalence of vascular events in hypertensive subjects. Age Ageing, 1999; 28: 23-28.

11. Brotman DJ, Davidson MB, Boumitri M, Vidt DG. Impaired diurnal blood pressure variation and all-cause mortality. Am J Hypertens, 2008; 21: 92-97.

12. Krzych LJ. Blood pressure variability in children with essential hypertension. J Hum Hypertens, 2007; 21: 494-500.

13. Fava $C$, Burri $\mathrm{P}$, Almgren $\mathrm{P}$ et al. Dipping and variability of blood pressure and heart rate at night are heritable traits. Am J Hypertens, 2005; 18: 1402-1407.

14. Wang X, Ding X, Su S et al. Genetic influences on daytime and night-time blood pressure: Similarities and differences. J Hypertens, 2009; 27: 2358-2364.

15. Cuspidi C, Meani S, Salerno M et al. Cardiovascular target organ damage in essential hypertensives with or without reproducible nocturnal fall in blood pressure. J Hypertens, 2004; 22: 273-280.

16. Cuspidi C, Meani S, Valerio C et al. Reproducibility of dipping/ /nondipping pattern in untreated essential hypertensive patients: Impact of sex and age. Blood Press Monit, 2007; 12: 101-106.

17. Omboni S, Parati G, Palatini P et al. Reproducibility and clinical value of nocturnal hypotension: Prospective evidence from the SAMPLE study. Study on Ambulatory Monitoring of Pressure and Lisinopril Evaluation. J Hypertens, 1998; 16: 733-738.

18. Hernández-del Rey R, Martin-Baranera M, Sobrino J et al. Reproducibility of the circadian blood pressure pattern in 24-h versus 48-h recordings: The Spanish Ambulatory Blood Pressure Monitoring Registry. J Hypertens, 2007; 25: 2406-2412. 
19. Stenehjem AE, Os I. Reproducibility of blood pressure variability, white-coat effect and dipping pattern in untreated, uncomplicated and newly diagnosed essential hypertension. Blood Press, 2004; 13: 214-224.

20. Manning G, Rushton L, Donnelly R, Millar-Craig MW. Variability of diurnal changes in ambulatory blood pressure and nocturnal dipping status in untreated hypertensive and normotensive subjects. Am J Hypertens, 2000; 13: 1035-1038.

21. Henskens LH, Kroon AA, van Oostenbrugge RJ, Haest RJ, Lodder J, de Leeuw PW. Different classifications of nocturnal blood pressure dipping affect the prevalence of dippers and non-dippers and the relation with target-organ damage. J Hypertens, 2008; 26: 691-698.

22. Verdecchia P, Angeli F, Borgioni C et al. Prognostic value of circadian blood pressure changes in relation to differing measures of day and night. J Am Soc Hypertens, 2008; 2: 88-96.

23. Hermida RC, Ayala DE, Mojón A, Fernández JR. Influence of circadian time of hypertension treatment on cardiovascular risk: Results of the MAPEC study. Chronobiol Int, 2010; 27: 1629-1651.

24. Takeda A, Toda T, Fujii T, Matsui N. Bedtime administration of long-acting antihypertensive drugs restores normal nocturnal blood pressure fall in nondippers with essential hypertension. Clin Exp Nephrol, 2009; 13: 467-472.

25. Hoshide Y, Kario K, Schwartz JE, Hoshide S, Pickering TG, Shimada K. Incomplete benefit of antihypertensive therapy on stroke reduction in older hypertensives with abnormal nocturnal blood pressure dipping (extreme-dippers and reverse-dippers). Am J Hypertens, 2002; 15: 844-850.

26. Kario K, Shimada K. Differential effects of amlodipine on ambulatory blood pressure in elderly hypertensive patients with different nocturnal reductions in blood pressure. Am J Hypertens, 1997; 10: 261-268.

27. Eguchi K, Kario K, Shimada K. Effects of long-acting ACE inhibitor (temocapril) and long-acting Ca channel blocker (amlodipine) on 24-h ambulatory BP in elderly hypertensive patients. J Hum Hypertens, 2001; 15: 643-648.

28. Qiu YG, Zhu JH, Tao QM et al. Captopril administered at night restores the diurnal blood pressure rhythm in adequately controlled, nondipping hypertensives. Cardiovasc Drugs Ther, 2005; 19: 189-195.

29. Fabbian F, Smolensky MH, Tiseo R, Pala M, Manfredini R, Portaluppi F. Dipper and non-dipper blood pressure 24-hour patterns: Circadian rhythm-dependent physiologic and pathophysiologic mechanisms. Chronobiol Int, 2012; doi: 10.3109/07420528.2012.715872.

30. Dauphinot V, Gosse P, Kossovsky MP et al. Autonomic nervous system activity is independently associated with the risk of shift in the non-dipper blood pressure pattern. Hypertens Res, 2010; 33: 1032-1037.

31. Kurpesa M, Trzos E, Drozdz J, Bednarkiewicz Z, Krzemińska-Pakuła M. Myocardial ischemia and autonomic activity in dippers and non-dippers with coronary artery disease: Assessment of normotensive and hypertensive patients. Int J Cardiol, 2002; 83: 133-142.

32. Abate G, D’Andrea L, Battestini M, Zito M, Di Iorio A. Autonomic nervous activity in elderly dipper and non-dipper patients with essential hypertension. Aging (Milano), 1997; 9: 408-414.

33. Grassi G, Seravalle G, Quarti-Trevano F et al. Adrenergic, metabolic, and reflex abnormalities in reverse and extreme dipper hypertensives. Hypertension, 2008; 52: 925-931.

34. Spallone V, Maiello MR, Cicconetti E et al. Factors determining the 24 -h blood pressure profile in normotensive patients with type 1 and type 2 diabetes. J Hum Hypertens, 2001; 15: 239-246.

35. Jermendy G, Ferenczi J, Hernandez E, Farkas K, Nadas J. Day-night blood pressure variation in normotensive and hypertensive NIDDM patients with asymptomatic autonomic neuropathy. Diabetes Res Clin Pract, 1996; 34: 107-114.
36. Hojo Y, Noma S, Ohki T, Nakajima H, Satoh Y. Autonomic nervous system activity in essential hypertension: A comparison between dippers and non-dippers. J Hum Hypertens, 1997; 11: 665-671.

37. Kario K, Mitsuhashi T, Shimada K. Neurohumoral characteristics of older hypertensive patients with abnormal nocturnal blood pressure dipping. Am J Hypertens, 2002; 15: 531-537.

38. Kario K, Schwartz JE, Pickering TG. Changes of nocturnal blood pressure dipping status in hypertensives by nighttime dosing of alpha-adrenergic blocker, doxazosin: Results from the HALT study. Hypertension, 2000; 35: 787-794.

39. Kario K, Schwartz JE, Pickering TG. Ambulatory physical activity as a determinant of diurnal blood pressure variation. Hypertension, 1999; 34: 685-691.

40. Cavelaars M, Tulen JH, van Bemmel JH, van den Meiracker AH. Physical activity, dipping and haemodynamics. J Hypertens, 2004; 22: 2303-2309.

41. Park S, Jastremski CA, Wallace JP. Time of day for exercise on blood pressure reduction in dipping and nondipping hypertension. J Hum Hypertens, 2005; 19: 597-605.

42. Shiotani H, Umegaki Y, Tanaka M, Kimura M, Ando H. Effects of aerobic exercise on the circadian rhythm of heart rate and blood pressure. Chronobiol Int, 2009; 26: 1636-1646.

43. Mansoor GA, White WB, McCabe EJ, Giacco S. The relationship of electronically monitored physical activity to blood pressure, heart rate, and the circadian blood pressure profile. Am J Hypertens, 2000; 13: 262-267.

44. Izdebska E, Cybulska I, Izdebskir J, Makowiecka-Ciesla M, Trzebski A. Effects of moderate physical training on blood pressure variability and hemodynamic pattern in mildly hypertensive subjects. J Physiol Pharmacol, 2004; 55: 713-724.

45. Narkiewicz K, Winnicki M, Schroeder K et al. Relationship between muscle sympathetic nerve activity and diurnal blood pressure profile. Hypertension, 2002; 39: 168-172.

46. Tsioufis C, Syrseloudis D, Dimitriadis K et al. Disturbed circadian blood pressure rhythm and C-reactive protein in essential hypertension. J Hum Hypertens, 2008; 22: 501-508

47. Maeda K, Yasunari K, Watanabe T, Nakamura M. Oxidative stress by peripheral blood mononuclear cells is increased in hypertensives with an extreme-dipper pattern and/or morning surge in blood pressure. Hypertens Res, 2005; 28: 755-761.

48. von Känel R, Jain S, Mills PJ et al. Relation of nocturnal blood pressure dipping to cellular adhesion, inflammation and hemostasis. J Hypertens, 2004; 22: 2087-2093.

49. Vyssoulis GP, Karpanou EA, Kyvelou SM et al. Nocturnal blood pressure fall and metabolic syndrome score in hypertensive patients. Blood Press Monit, 2007; 12: 351-356.

50. Okamoto LE, Gamboa A, Shibao C et al. Nocturnal blood pressure dipping in the hypertension of autonomic failure. Hypertension, 2009; 53: 363-369.

51. Ukkola O, Vasunta RL, Kesäniemi YA. Non-dipping pattern in ambulatory blood pressure monitoring is associated with metabolic abnormalities in a random sample of middle-aged subjects. Hypertens Res, 2009; 32: 1022-1027.

52. Pistrosch F, Reissmann E, Wildbrett J, Koehler C, Hanefeld M. Relationship between diurnal blood pressure variation and diurnal blood glucose levels in type 2 diabetic patients. Am J Hypertens, 2007; 20: 541-545.

53. Diamantopoulos EJ, Andreadis E, Tsourous G et al. Insulin resistance and blood pressure circadian variation in an obese hypertensive population. Clin Exp Hypertens, 2006; 28: 625-630.

54. Fagard RH, Thijs L, Staessen JA et al. Prognostic significance of ambulatory blood pressure in hypertensive patients with history of cardiovascular disease. Blood Press Monit, 2008; 13: 325-332.

55. Fagard RH, Thijs L, Staessen JA et al. Night-day blood pressure ratio and dipping pattern as predictors of death and cardiovascular events in hypertension. J Hum Hypertens, 2009; 23: 645-653. 
56. Fagard RH, Celis H, Thijs L et al. Daytime and nighttime blood pressure as predictors of death and cause-specific cardiovascular events in hypertension. Hypertension, 2008; 51: 55-61.

57. Li Y, Boggia J, Thijs L et al. Is blood pressure during the night more predictive of cardiovascular outcome than during the day? Blood Press Monit, 2008; 13: 145-147.

58. Boggia J, Li Y, Thijs L et al. Prognostic accuracy of day versus night ambulatory blood pressure: A cohort study. Lancet, 2007; 370: 1219-1229.

59. Pringle E, Phillips C, Thijs L et al. Systolic blood pressure variability as a risk factor for stroke and cardiovascular mortality in the elderly hypertensive population. J Hypertens, 2003; 21: 2251-2257.

60. Tripepi G, Fagugli RM, Dattolo $\mathrm{P}$ et al. Prognostic value of 24-hour ambulatory blood pressure monitoring and of night/ /day ratio in nondiabetic, cardiovascular events-free hemodialysis patients. Kidney Int, 2005; 68: 1294-1302.

61. Ishikawa J, Shimizu M, Hoshide S et al. Cardiovascular risks of dipping status and chronic kidney disease in elderly Japanese hypertensive patients. J Clin Hypertens (Greenwich), 2008; 10: 787-794.

62. Rahman M, Griffin V, Heyka R, Hoit B. Diurnal variation of blood pressure; reproducibility and association with left ventricular hypertrophy in hemodialysis patients. Blood Press Monit, 2005; 10: 25-32.

63. Hermida RC, Ayala DE, Mojón A, Fernández JR. Decreasing sleep-time blood pressure determined by ambulatory monitoring reduces cardiovascular risk. J Am Coll Cardiol, 2011; 58: 1165-1173.

64. Verdecchia P, Schillaci G, Borgioni C et al. Gender, day-night blood pressure changes, and left ventricular mass in essential hypertension. Dippers and peakers. Am J Hypertens, 1995; 8: 193-196.

65. Cuspidi C, Macca G, Sampieri L et al. Target organ damage and non-dipping pattern defined by two sessions of ambulatory blood pressure monitoring in recently diagnosed essential hypertensive patients. J Hypertens, 2001; 19: 1539-1545.

66. Amici A, Cicconetti P, Sagrafoli C et al. Exaggerated morning blood pressure surge and cardiovascular events. A 5-year longitudinal study in normotensive and well-controlled hypertensive elderly. Arch Gerontol Geriatr, 2009; 49: e105-e109.

67. Cuspidi C, Meani S, Salerno M et al. Cardiovascular target organ damage in essential hypertensives with or without reproducible nocturnal fall in blood pressure. J Hypertens, 2004; 22: 273-280.

68. Ivanovic BA, Tadic MV, Celic VP. To dip or not to dip? The unique relationship between different blood pressure patterns and cardiac function and structure. J Hum Hypertens, 2011; doi: 10.1038/jhh.2011.83.

69. Nakatsu T, Shinohata R, Mashima K et al. Use of plasma B-type natriuretic peptide level to identify asymptomatic hypertensive patients with abnormal diurnal blood pressure variation profiles: Nondippers, extreme dippers, and risers. Hypertens Res, 2007; 30: 651-658.

70. Marinakis AG, Vyssoulis GP, Michaelides AP, Karpanou EA, Cokkinos DV, Toutouzas PK. Impact of abnormal nocturnal blood pressure fall on vascular function. Am J Hypertens, 2003; 16: 209-213.

71. Bellelli G, Frisoni GB, Lucchi E et al. Blunted reduction in nighttime blood pressure is associated with cognitive deterioration in subjects with long-standing hypertension. Blood Press Monit, 2004; 9: 71-76.

72. Guo H, Tabara Y, Igase M et al. Abnormal nocturnal blood pressure profile is associated with mild cognitive impairment in the elderly: The J-SHIPP study. Hypertens Res, 2010; 33: 32-36.
73. Muxfeldt ES, Cardoso CR, Salles GF. Prognostic value of nocturnal blood pressure reduction in resistant hypertension. Arch Intern Med, 2009; 169: 874-880.

74. Bouhanick B, Bongard V, Amar J, Bousquel S, Chamontin B. Prognostic value of nocturnal blood pressure and reverse-dipping status on the occurrence of cardiovascular events in hypertensive diabetic patients. Diabetes Metab, 2008; 34: 560-567.

75. Eto M, Toba K, Akishita M et al. Impact of blood pressure variability on cardiovascular events in elderly patients with hypertension. Hypertens Res, 2005; 28: 1-7.

76. Pandian JD, Wong AA, Lincoln DJ et al. Circadian blood pressure variation after acute stroke. J Clin Neurosci, 2006; 13: 558-562.

77. Bastos JM, Bertoquini S, Silva JA, Polónia J. Relationship between ambulatory blood pressure monitoring values and future occurrence of ischemic cerebrovascular and coronary events in hypertensive patients. Rev Port Cardiol, 2006; 25: 305-316.

78. Eguchi K, Pickering TG, Hoshide S et al. Ambulatory blood pressure is a better marker than clinic blood pressure in predicting cardiovascular events in patients with/without type 2 diabetes. Am J Hypertens, 2008; 21: 443-450.

79. Astrup AS, Nielsen FS, Rossing P et al. Predictors of mortality in patients with type 2 diabetes with or without diabetic nephropathy: A follow-up study. J Hypertens, 2007; 25: 2479-2485.

80. Sturrock ND, George E, Pound N, Stevenson J, Peck GM, Sowter H. Non-dipping circadian blood pressure and renal impairment are associated with increased mortality in diabetes mellitus. Diabet Med, 2000; 17: 360-364.

81. Shin J, Kline S, Moore M et al. Association of diurnal blood pressure pattern with risk of hospitalization or death in men with heart failure. J Card Fail, 2007; 13: 656-662.

82. Ingelsson E, Björklund-Bodegard K, Lind L, Arnlöv J, Sundström J. Diurnal blood pressure pattern and risk of congestive heart failure. JAMA, 2006; 295: 2859-2866.

83. Pierdomenico SD, Lapenna D, Cuccurullo F. Risk of atrial fibrillation in dipper and nondipper sustained hypertensive patients. Blood Press Monit, 2008; 13: 193-197.

84. Ohkubo T, Imai Y, Tsuji I et al. Relation between nocturnal decline in blood pressure and mortality. The Ohasama Study. Am J Hypertens, 1997; 10: 1201-1207.

85. Mancia G, Bombelli M, Facchetti R et al. Long-term prognostic value of blood pressure variability in the general population: Results of the Pressioni Arteriose Monitorate e Loro Associazioni Study. Hypertension, 2007; 49: 1265-1270.

86. Hansen TW, Thijs L, Li Y et al. Prognostic value of reading-to-reading blood pressure variability over 24 hours in 8938 subjects from 11 populations. Hypertension, 2010; 55: 1049-1057.

87. Hansen TW, Li Y, Boggia J, Thijs L, Richart T, Staessen JA. Predictive role of the nighttime blood pressure. Hypertension, 2011; 57: 3-10.

88. Gavish B, Ben-Dov IZ, Kark JD, Mekler J, Bursztyn M. The association of a simple blood pressure-independent parameter derived from ambulatory blood pressure variability with shortterm mortality. Hypertens Res, 2009; 32: 488-495.

89. Yano Y, Kario K. Nocturnal blood pressure, morning blood pressure surge, and cerebrovascular events. Curr Hypertens Rep, 2012; 14: 219-227.

90. Yano Y, Kario K. Nocturnal blood pressure and cardiovascular disease: A review of recent advances. Hypertens Res, 2012; 35 : 695-701. 